Special issue of the 3rd International Conference on Computational and Experimental Science and Engineering (ICCESEN 2016)

\title{
A Prediction Study on Bremsstrahlung Photon Flux of Tungsten as a Radiological Anode Material by using MCNPX and ANN Modeling
}

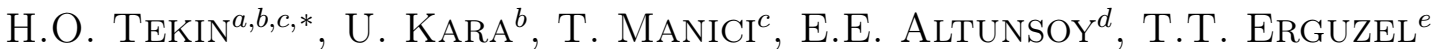 \\ ${ }^{a}$ Uskudar University, Vocational School of Health Services, Radiotherapy Department, Istanbul, Turkey \\ ${ }^{b}$ Suleyman Demirel University, Vocational School of Health Service, Medical Imaging Department, Isparta, Turkey \\ ${ }^{c}$ Uskudar University, Medical Radiation Research Center (USMERA), Istanbul, Turkey \\ ${ }^{d}$ Uskudar University, Vocational School of Health Services, Medical Imaging Department, Istanbul, Turkey \\ ${ }^{e}$ Uskudar University, Faculty of Engineering and Natural Sciences, Computer Engineering, Istanbul, Turkey
}

Medical imaging is a technique that is mostly known as visual representations of the parts of body for clinical scans and analysis. In imaging process for medical purpose there take part radiologists, radiographers/radiology technicians, medical physicists, sonographers, nurses, and engineers. As an apart issue from the medical imaging devices, we can treat X-rays using devices such as radiography, computed tomography, fluoroscopy, dental conebeam computed tomography, and mammography. All these devices are to perform X-ray using during medical imaging process. An X-ray beam is generated in a vacuum tube that is principally composed of an anode and a cathode material to produce X-ray beams, whose name is X-ray tube. The anode represents the component in which the X-ray beam produced that made from a piece of metal. For decades, tungsten (W) has been used as an anode material of various X-ray tubes. Tungsten has high atomic number and high melting point of $3370^{\circ} \mathrm{C}$ with low rate of volatilization. In this study, we performed Monte Carlo simulation for flux calculations of $\mathrm{W}$ target by using MCNP-X general purpose code and considered result as a data set for artificial neural network. It can be concluded that the results agreed well between Monte Carlo simulation and artificial neural network prediction.

DOI: 10.12693/APhysPolA.132.433

PACS/topics: artificial neural network, Monte Carlo, medical imaging

\section{Introduction}

The simulation techniques of generated X-ray spectra are one of the most significant tools for different kind of investigations, especially on patient absorbed dose and obtained image quality in diagnostic radiology. The Monte Carlo analysis in the radiological investigations has been used for several decades. To estimate the absorbed dose by different tissues and different organs in the patient body is significantly needed to determine the risks due to the absorbed radiation so that diagnostic technique can be properly justified [1].

$\mathrm{X}$-rays are the main tools in the diagnostic radiology. Knowing the main important parameters of the generated X-rays can allow us to determine the basic diagnostic requirements such as image quality, exposure dose etc. and it should be always in investigation [2].

Nowadays, studies on generated X-rays are rapidly growing by using different methods. One of those methods are Monte Carlo (MC) method. Monte Carlo method is a numerical test to simulate an event by using the numbers between 0 and 1. By using the Monte Carlo method, it is possible to optimize the experimental tools used before the experiments. In addition, this method is widely

\footnotetext{
* corresponding author; e-mail:

huseyinozan.tekin@uskudar.edu.tr
}

used in the field of medical imaging and the particle detectors in nuclear physics studies. Monte Carlo method is a strong tool not only in the field of optimising the different kinds of devices but also the different radiation kinds and their interactions with matter.

In recent years, MC method is widely used to simulate interaction of medical radiation with tissues and environment. MCNPX is a strong tool for various kind of applications. The features of MCNPX code has been investigated in literature. Different kinds of applications by using MCNPX code have been studied by different authors. An investigation on abilities of MCNPX on detector efficiency and radiation attenuation properties has been studied by Tekin [3]. The capability of MCNPX Monte Carlo code on detection efficiency and usage of different experimental and Monte Carlo studies has been studied by Akkurt et al. [4]. Also using standards of MCNPX for dose distribution in PET-CT facility have been studied by Tekin and Kara [5]. In addition, some other MC studies have been found in literature [6-10]. In this study, we performed MC simulation for flux calculations of W target by using MCNPX general purpose code and considered result as a data set for artificial neural network $(\mathrm{ANN})$.

\section{Materials and methods}

Artificial neural network is an information process which is inspired by the system of human brain such as brain information network [11]. ANN includes some sig- 
nificant parts such as input layer, one or more hidden layers and output layer, respectively. The basic structure of ANN is shown in Fig. 1. The input and output data are considered as information processing systems that have the abilities to learn, recall and generalize from sample training data.

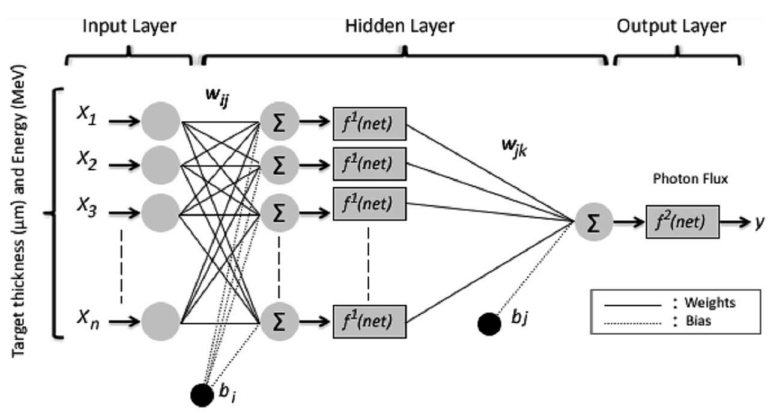

Fig. 1. Structure of artificial neural network used in the model.

As a Monte Carlo code, MCNPX (version 2.6.0) has been considered. MCNPX is a general purpose radiation transport code for modeling the interaction of radiation with materials and also tracks all particles at wide range of energies. MCNPX is fully three-dimensional and it utilizes extended nuclear cross-section libraries and uses physics models for particle types [12]. MCNPX is a strong tool for various kind of applications. Energy source in input file has been defined as $10 \mathrm{MeV}$ monoenergetic electron-beam. The data card section of MCNPX input has been defined by considering different variable such as CEL, ERG, DIR, POS, and PAR. The geometric center of detection cell has been considered for location of point source. Each variable has different abilities during the Monte Carlo simulation.

In this study, we obtained the bremsstrahlung photon flux from the $\mathrm{W}$ target by using MCNPX MC code by selecting the commanded source cell, energy, direction, source position, and particle types as our variables. The obtained result has been considered as input parameter to ANN model. The input parameters have been selected as target thickness and electron beam energy in ANN model.

\section{Result and discussion}

Radiology and its equipment features and parameters during the diagnostic process have important places not only for well medical images but also one of the most important radiation protection principle namely ALARA. To investigate the anode materials is the one of main research filed in diagnostic radiology. We considered $\mathrm{W}$ target as a target material with $2 \mu \mathrm{m}$ thickness. Tungsten is a metal that can be used in most of X-ray tubes as an anode material. For decades, $\mathrm{W}$ has been used as an anode material of various X-ray tubes because of different kind of properties. Tungsten has high atomic number and high melting point of $3370^{\circ} \mathrm{C}$ with low rate of volatilization. This all features makes $\mathrm{W}$ an ideal anode material in medical imaging process. As a first step of this study, $10 \mathrm{MeV}$ mono-energetic electron beam has been directed onto $\mathrm{W}$ target material. Calculated photon fluence sprectra from $\mathrm{W}$ target material is shown in Fig. 2. During the generation of training data, we used the $80 \%$ of obtained photon flux Monte Carlo data which has been obtained from MCNPX. Afterwards, we used the rest, $20 \%$ of data obtained from the same Monte Carlo simulation as a test data for evaluation of the predictions. We considered $\mathrm{W}$ target as a target material with $2,4,6,8,10 \mu \mathrm{m}$ thicknesses.

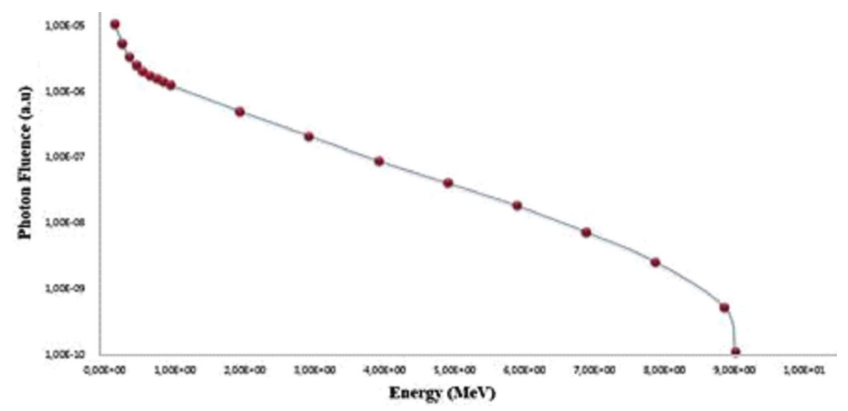

Fig. 2. Obtained bremsstrahlung photon flux by MCNPX.

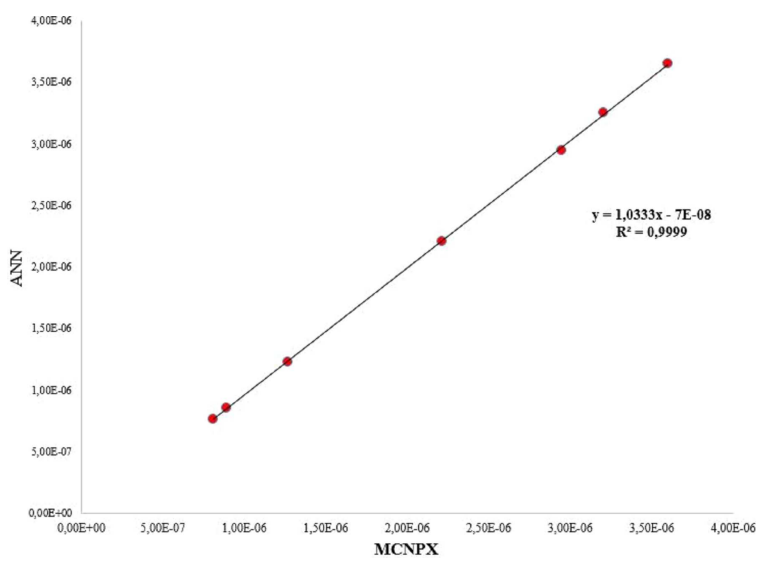

Fig. 3. Correlation between ANN and MCNPX.

It can be clearly seen in Fig. 3 that a good correlation has been obtained between calculated MCNPX and estimated ANN data. By considering the method of recent study, some similar studies have been found in literature. Investigation of correlation of EGSnrc and ANN model has been studied by Akkurt et al. [13] and a good agreement has been obtained.

\section{Conclusions}

It can be concluded that MCNPX is a strong and effective tool for investigations on bremsstrahlung photon flux where experimental results are not available. Nowadays, radiological anode materials as investigation subject have 
a special place especially for new generation radiological devices. It can be also concluded that investigated standard MCNPX geometry can be used for potential future studies since nanotechnology is also recently used for production of various radiation shields and technologies. On the other hand, it can be concluded that ANN is a strong prediction model and can be used for different future investigations. Some other statistical methods considering linear and nonlinear relations could also be used in order to compare the aforementioned methods.

\section{References}

[1] E. Massoud, H.M. Diab, J. Cell Sci. Ther. 5, 155 (2014).

[2] U. Kara, H.O. Tekin, Conf. Proc. 1, 195 (2016).

[3] H.O. Tekin, Sci. Technol., Nucl. Install. 2016, 6547318 (2016).

[4] I. Akkurt, H.O. Tekin, A. Mesbahi, Acta Phys. Pol. A 128, B-332 (2015).
[5] H.O. Tekin, U. Kara, J. Commun. Comput. 13, 32 (2016).

[6] U. Kara, A. Mesbahi, I. Akkurt, Acta Phys. Pol. A 128, B-378 (2015).

[7] N. Demir, Z.N. Kuluozturk, I. Akkurt, Acta Phys. Pol. A 128, B-443 (2015).

[8] H.O. Tekin, V.P. Singh, T. Manici, J. Polytechn. 19, 617 (2016).

[9] H.O. Tekin, V.P. Singh, T. Manici, Appl. Radiat. Isotop. 121, 122 (2017).

[10] H.O. Tekin, T. Manici, Nucl. Sci. Techn. 28, 95 (2017).

[11] J. Schmidhuber, Neural Networks 61, 85 (2015).

[12] RSICC Computer Code Collection, MCNPX User's Manual, Version 2.4.0., (2002), Monte Carlo NParticle Transport Code System for Multiple and High Energy Applications.

[13] I. Akkurt, K. Gunoglu, H.O. Tekin, Z.N. Demirci, G. Yegin, N. Demir, Iran. J. Rad. Res. 10, 63 (2011). 